Ophthalmologica 1947;114:331

\title{
Über Sitz und Optik der Kontaktschalen
}

\begin{tabular}{|l|l|l}
\hline O. & Knüsel \\
\hline
\end{tabular}

Aarau

Manuskript nicht eingegangen

Diskussion

R. Dufour (Lausanne): Suivant les conseils de Magitot et de Paufique nous commençons à la clinique ophtalmique de Lausanne

a prescrire des verres de contact de Dudragne à Paris

d'après moulage de $\Gamma$ ceil au Négocol et verres d'essai de Dudragne. 